\title{
EFFECTIVE IMAGE MODELS FOR INSPECTING PROFILE FLAWS OF CAR MIRRORS WITH APPLICATIONS
}

\author{
Yuan-Shyi Peter Chiu, Yu-Kai Lin, Hong-Dar Lin* \\ Chaoyang University of Technology, Taichung, Taiwan
}

Since car mirrors are standard accessories with cars, the demand for car mirrors is growing and manufacturers also pay more emphasis on the increase of product quality. Common appearance flaws of car mirrors include: scratches, bubbles, pinholes causing surface flaw type and burrs, damaged edges causing profile flaw type. Currently, the inspection tasks are conducted by human inspectors. Since the profile flaws will cause structural damages of car mirrors and reduce ability to withstand stress, the level of damage suffered even more than the surface flaws. In addition, the angle diversity of capturing images makes it hard to implement automatic optical inspection. Therefore, this study develops an automated profile flaw detection system for car mirrors to replace visual inspection personnel. This study proposes a self-contrast defect detection method for the profile flaw inspection of car mirrors. It is not required to provide a standard flawless sample in detection process and derive information compared with testing samples. The proposed method first extracts the contour information of the testing image by Fourier descriptors. Then, after some middle and high-frequency coefficients were filtered out, an approximated contour image can be rebuilt from the Fourier domain for comparing with the testing image. Finally, the flaw districts are easily separated by image subtraction. Experimental results demonstrate that the flaw inspection rate reaches $85.05 \%$, and the incorrect alert rate is smaller than $0.07 \%$, and the correct classification rate is up to $97.47 \%$.

Key words: car mirrors, computer vision, profile flaws, fourier descriptors

\section{INTRODUCTION}

Glass-related goods have turned into essential things in our daily routine and main materials for architecture and manufacturing industries. There are some typical applications of glass and mirrors in our daily life, such as car mirrors, building windows, artifacts, LCD panels, etc. Because appearance defects influence the qualityof the mirrors, the detection of appearance defects is very vital. In the manufacturing process of mirror-related goods, a metal is coated on the rear of see-through glass. Manual inspection is easy to be disturbed by the outer object images reflected on the surfaces of mirrors and leads to wrong decisions. Furthermore, the exterior of a mirror is easily fastened to dust, dirt, water,etc. and make the flaw inspection harder. Figure 1 shows a common car mirror set including two side-view mirrors and one rearview mirror.

Since car mirrors are made of curve glass with the aluminum- or chromium-coated materials, this makes mirrors have high reflectance. Common appearance defects of car mirrors include: scratches, bubbles, pinholes causing surface defect type; and damaged edges, burrs causing profile defect type. After proceeding the grinding operation, the burr defects almost do not exist. The damaged edges are the primary flaws in the profile defect type. The defect sizes of car mirror need to be inspected out are at least $0.20 \mathrm{~mm}$ for the surface flaw type and 0.26 $\mathrm{mm}$ for the profile flaw type. Figure 2 shows a work-inprocess (WIP) mirror with two types of surface flaw and profile flaw. Appearance flaws on the curved surfaces are difficult to be examined for technical inspectors due

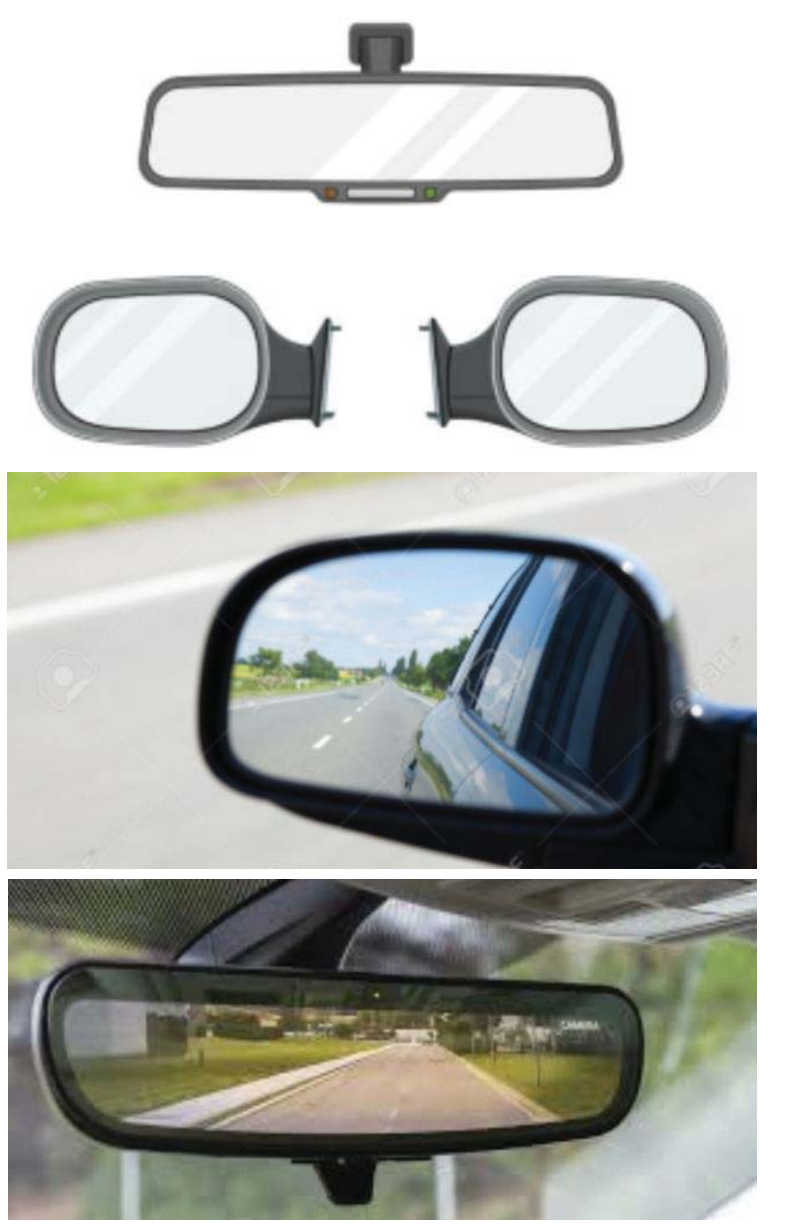

(a)

(b)

Figure 1: Common car mirrors: (a) a car mirror set, (b) a side-view mirror, and (c) a rearview mirror 


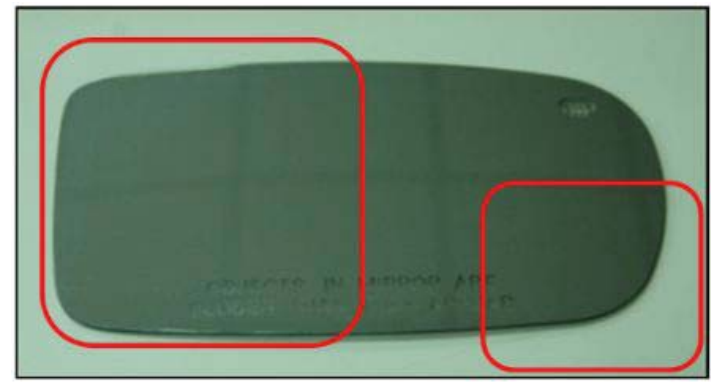

(a)

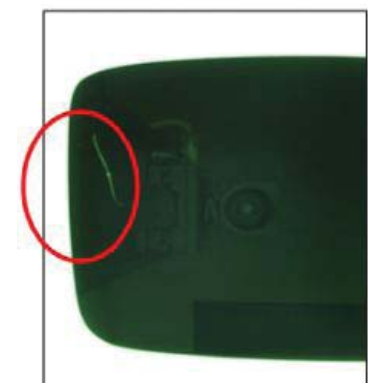

(b)

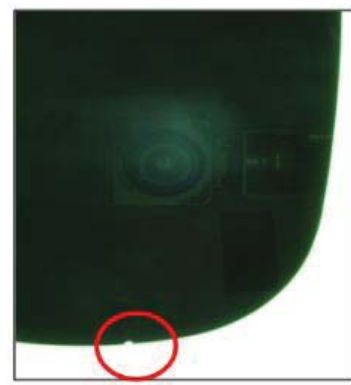

(c)

Figure 2: (a) A WIP mirror with two types of (b) the surface flaw and (c) the profile flaw
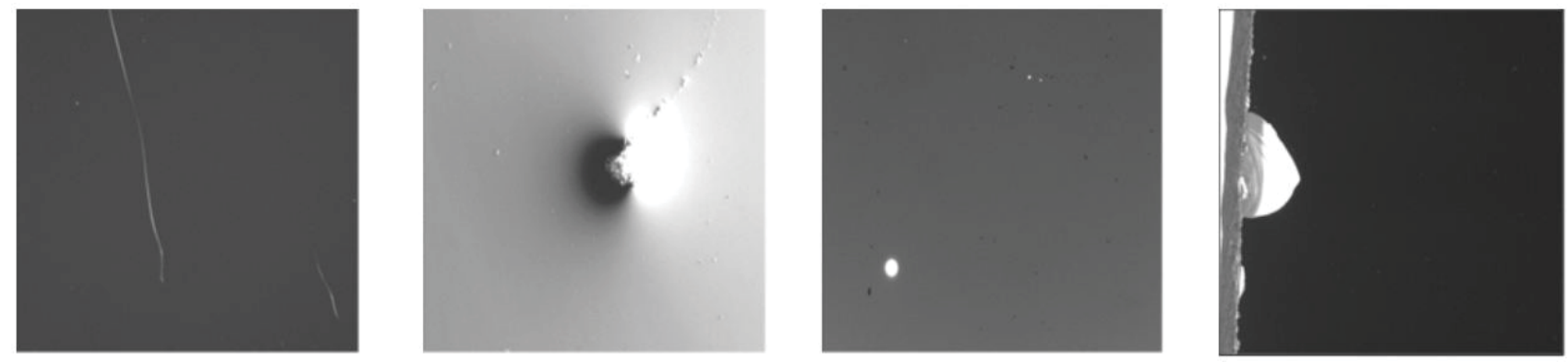

Figure 3: Four regular defect kinds of car mirrors: (a) scratch, (b) bubble, (c) pinhole, (d) damaged edge

Table 1: Comparison table of the two defect types of car mirrors

\begin{tabular}{|c|c|c|}
\hline Types & Surface defects & Profile defects \\
\hline Defect kinds & scratch, bubble, pinhole & damaged edge, burr \\
\hline Locations & on surfaces of mirrors & on contours of mirrors \\
\hline Properties & different gray levels & shape change of a mirror contour \\
\hline Methods & detection of gray level changes & shape matching with a template \\
\hline
\end{tabular}

to light reflection on mirror surfaces. Figure 3 displays four regular defect kinds of car mirrors: (a) scratch, (b) bubble, (c) pinhole, and (d) damaged edge.

Appearance flaws can be classified into two categories, surface flaw and profile flaw. Comparing the properties of surface defects and profile defects, the theories of detection methods are significantly different. The methods detecting surface defects are based on the changes of gray levels and the others detecting profile defects are based on the shape matching of an object contour. Table 1 lists a comparison table of the two defect types of car mirrors. Since the profile defects will cause structural damages of car mirrors and reduce the ability to withstand stress, the degree of harm even more than the surface defects for car mirrors.

Car mirrors are being manufactured in curve shapesfor obtaining wider field of views, which increases difficulties of inspecting the products. The most usual inspection scheme for surface flaws on car mirrors is manual inspection by human eyes. Mirror inspection needs particular working environment, specifically with respect to lighting. In the practical application, every testingmirror is carried into the assessor's field of view. A car mirror has a rectangle shape with round corners and curved surface andthe profile flaws to be examined could be found on the outer edges of the mirror. The curved mirror has the appearance of higher reflectance and wider field of view which is more complex than that of the plane mirror. It is harder to recognize profile flaws embedded on the curved surfaces. The majority of flaws have many kinds and can suppose different forms. Figure 4 demonstrates the inspection procedure of car mirrors in production site.

Manual visual inspection is tedious, laborious and extremely relied upon the examiners' skills. Mistaken judgements are simply made since examiners' eye tiredness and subjectivity. Since the car mirror has the appearance of high reflection, those reflected lightings make the flaw detection task more difficult when profile flaws are inlaid on the curved surfaces of car mirrors. High reflection on curved mirrors increases the difficulty of distinguishing the profile flaws on car mirrors. In this study, the trial WIP mirrors with length $18.1 \mathrm{~cm}$, width $10.71 \mathrm{~cm}$, and thickness $0.2 \mathrm{~cm}$, were arbitrarily chosen from the production line of car mirrors. Figure 5 shows the scale of a trial 


\begin{tabular}{|c|c|c|}
\hline $\begin{array}{c}\text { (1) Wear gloves before } \\
\text { inspecting mirrors }\end{array}$ & (2) Wipe mirrors with a rag & $\begin{array}{c}\text { (3) Inspect mirrors while } \\
\text { close to light source }\end{array}$ \\
\hline $\begin{array}{c}\text { (4) Mark defect locations on } \\
\text { mirrors }\end{array}$ & $\begin{array}{c}\text { (5) Separate normal and } \\
\text { defective mirrors }\end{array}$ & $\begin{array}{c}\text { (6) Count numbers of various } \\
\text { defective items }\end{array}$ \\
\hline & & \\
\hline
\end{tabular}

Figure 4: Inspection procedures for car mirrors

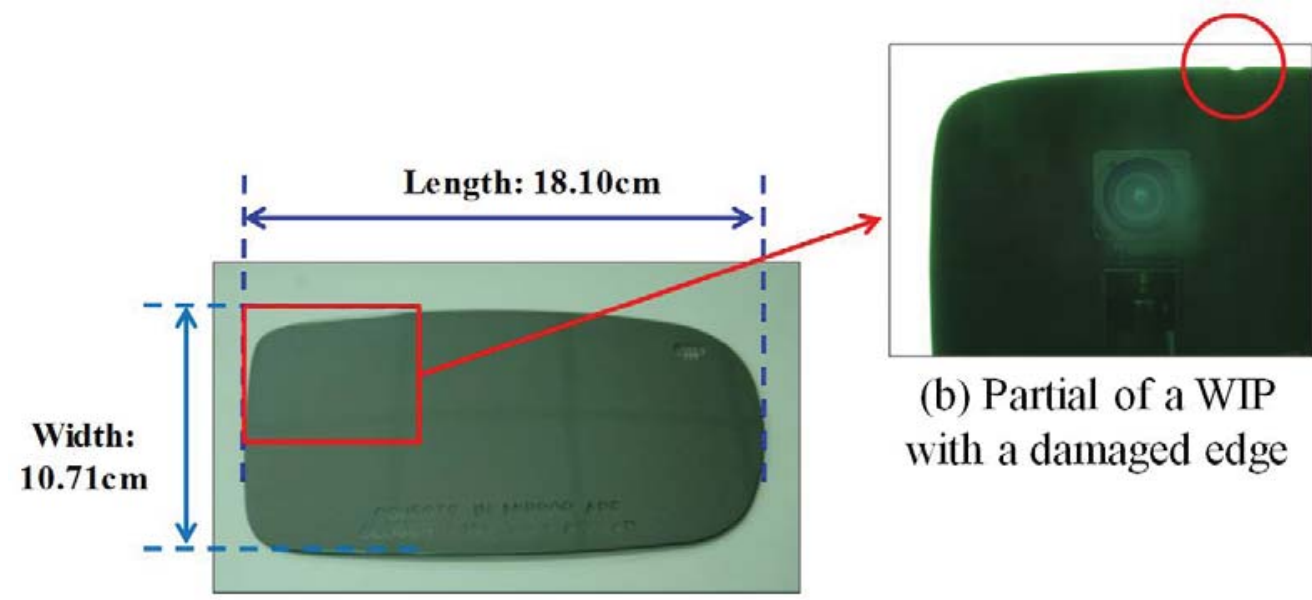

(a) Dimensions of a WIP

Figure 5: The scale of a trial WIP mirror with a flaw of damaged edge

WIP mirror (Fig. 5(a)) and a partial of the WIP with a flaw of damaged edge (Fig. 5(b)). The angle diversity of capturing images makes it hard to implement automated defect inspection. Consequently, this study establishes an automated profile defect inspection system of car mirrors to replace visual inspection personnel from car mirror inspection tasks. We suggest a Fourier descriptors based scheme to examine profile flaws on curved mirrors of vehicles.

The rest of the paper is composed as follows: Firstly, the articles on existing techniques of image processing for blemish inspection are reviewed. Secondly, the suggested image models for detecting flaws on car mirrors are described. Thirdly, the trials are conducted and the manifestation of the suggested models with traditional techniquesare evaluated. Finally, the contributions are summarized and the further workis indicated.

\section{LITERATURE REVIEW}

Automatic detection of appearance flaws has turned into a crucial work for producers striving to upgrade goods quality and manufacturing efficiency [1-2]. Lin and Chiu [3] developed a cosine transform based image rebuilt scheme for automatic inspection of little surface variations on capacitor chips of passive components. Lin et al. [4] applied the convolutional neural network with deep learning to conduct automatic inspection of light-emitting diode chips. Park et al. [5] constructed and examined many kinds of deep networks of various depths and layer nodes to choose suitable architecture for automatic detection of dirt, scratches, burrs, and wears on textured and non-textured surface parts.

Numerous research examined the visual flaw detection of glass-related goods. Li et al. [6] applied the principal components analysis approach to inspect surface defects on the mobile phone cover glass. Lin and Tsai [7] presented a Fourier transform-based method to inspect linear flaws on surfaces of capacitive touch displays. Chiu and Lin [8] developed a defect detection system with wavelet transformation based filtering scheme for automated inspection of area flaws on appearances of capacitive touch panels. Lin and Chiu [9] combined block cosine transform, Hotelling's distance measure, and grey 
theory to detect appearance flaws on clear LED lenses. Lin and Chen [10] combined the wavelet packet transform and the partial least squares technique to detect appearance flaws on textured LED lenses.

Some studies further focused on investigating the visual blemish inspection of car mirror products. Chiu et al. [11] addressed a Hough transform method for visual distortion blemish inspection on transparent glass of car mirrors. Lin and Hsieh [12] developed an optical inspection system based on small deviation control skills to find appearance variations on curved car mirrors. From the above reviews of literatures, almost all of the current researches focus on industrial inspections of LED lenses, transparent glass, and mirrors. These visual inspection systems concentrate mainly on the surface and distortion flaw detection. Since the profile defects will cause structural damages of products and reduce ability to with stand stress, the level of damage suffered even more than the surface flaws. These researches do not inspect profile flaws with properties of little flaws on curved surfaces of car mirrors. Accordingly, this study applies Fourier descriptors to profile flaw inspection on curved surfaces of car mirrors with high reflection.

Information of shape is conveyed through changes in the slopes of an object's boundary. The greatest change of the slopes is also called the largest curvature. Corner detection is identical to find the high curvature points on planar curve. Corners play an important role in shape perception by human and object description by computer vision system. Many corner detection methods calculated curvature maximal points using $\mathrm{k}$-cosines as corners [13]. They are applied to damage detection in civil engineering $[14,15]$ and defect detection in manufacturing industry [16]. Shapes of objects are depicted by their schematic curves. Matching typically involves finding a mapping from one curve to the other that minimizes allowable deviations. The curvature-based methods generally suffer from shortage of rotation, translation, and scaling invariance, sensitivity to object deformations, and need referential images for template matching.

Fourier descriptors are one of the highly employed shape expression schemes. The Fourier descriptors indicate the shape of an object in a frequency domain. The primary thought of a Fourier descriptor is to utilize the Fourier transformed borders as the shape features. Khoje and Bodhe [17] compared classification performance of the Fourier transform based shape descriptors and its derivatives for fruit quality grading of mangoes. Burla et al. [18] developed two modified Fourier descriptors methods for fast detection of surface defects on microlens arrays, one kind of micro-optical elements. Zheng et al. [19] introduced a revised Fourier descriptors based on multiscale centroid contour distance to recognize objects in remote sensing images. The theory of this approach is straightforward and computational efficiency.

The Fourier descriptors method supplies a computationally efficient option with sufficient frequency resolu- tion. In the Fourier transform, the filtering operations are used to the low-frequency coefficients. Thus, the Fourier descriptors have excellent compressibility to provide the function by using a few low-frequency components from the input shape information to describe and rebuild the approximate shape boundary [20]. In addition, the Fourier descriptors are simple to normalize data and are invariant to shape's rotation, scaling, translation, and changes of different starting points of boundary [21, 22]. This Fourier descriptors scheme can achieve self-comparison between the testing image and the rebuilt one and do not need referential images and template matching processes [23]. Derivatives of the Fourier descriptors as shape descriptors improved the shape classification and retrival performance through combining with wavelet transform and multiple resolutions [24].

\section{MATERIALS AND METHODS}

This study proposes a self-contrast flaw detection approach for the profile defect inspection of car mirrors. The self-comparison means it is not required to provide a standard flawless sample in detection process and derive information compared with the testing images. The inspection task of this research comprises discovering profile flaws on curved surfaces of car mirrors. Many of these unforeseen flaws are diverse with various forms and cannot be depicted by clear measures, thereby making automatic flaw inspection hard. Five stages are developed to accomplish the procedure of the profile flaw inspection. First, image preprocessing is carried out to eliminate background district and generate a binary edge image of objects by applying the Otsu method [25] for object separation from background and Sobel edge detector [13] for edge detection to obtain the edge information of the objects. Second, the binary edge image is converted to Fourier domain and the contour information of the testing image are extracted by Fourier descriptors. Third, by selecting the proper retaining rate in frequency domain, the low-frequency components are recovered and the others are assigned to zero for rebuilding the object contour. There is essential information in the retained low-frequency parts than those in the high-frequency parts. Fourth, the filtered frequency components are conducted by the reverse 1-D Fourier transform to produce a rebuilt image. Then, an approximated contour image can be rebuilt from the Fourier domain for comparing with the original image as a reference pattern. Fifth, the rebuilt image deducts with primitive testing image to obtain the difference image where the profile flaws have been detected. Therefore, the profile flaws on the car mirrors with curved surfaces will be correctly detected and recognized by the suggested approach. Figure 6 describes the research procedure of the proposed approach. It represents that the Fourier descriptors have the merits of being fine enough to extract required information from the retained components and indicating the abstraction of object shapes. 


\section{IMAGE MODELING\& ANALYSIS}

For obtaining better image quality and defect detection performance, a testing sample is divided into 6 parts for image acquisition. Thus, an entire testing sample is equally captured into 6 testing images to clearly display the details of object contours on each image (Figure 7). This study uses Otsu method to binarize the testing images for separating the objects from the background and then apply Sobel edge detector to the binary images for obtaining the edge information of the objects. Figure 8 shows the results in process of image binarization and edge detection: (a) primitive image, (b) binary image, (c) edge image. The edge information of objects on each image are clearly recorded.

Shape is a main measure for comparing objects based on their contours and physical structures. Fourier descriptors (FDs) offer a means of depicting the pattern of a 2-D closed form by conducting the Fourier transform of the border, where every $(x, y)$ point on the border is mapped to a complex number $x+y i$. The primitive shape can be rebuilt by taking the reverse Fourier transform. The low-frequency coefficients in Fourier domain are usually the most significant and are applied to obtain shape descriptors. Shape descriptors utilizing Fourier transformed components are invariant to variations of shape borders. A conventional method to deal with FDs is to let the descriptors correlated to values higher than a given frequency to zero and then reconstruct the shape. The influence of this operation is to smoothen the object's border, that is a low-pass filtering of the shape. Because

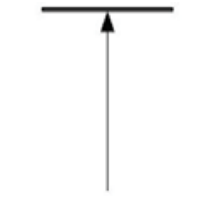

Image preprocessing

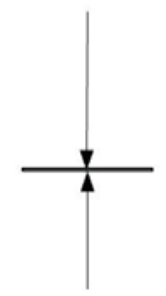

Shape rebuilt of objects in frequency

domain

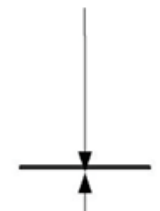

Comparison of image differences

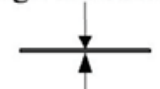

Evaluation

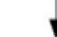

Figure 6: Research procedure of the proposed method
Input testing image

Binarize the image by Otsu method

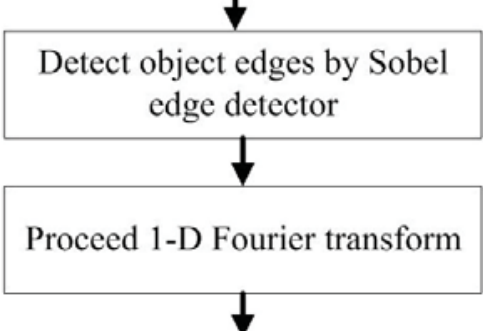

Select retaining rate and recover low-frequency components

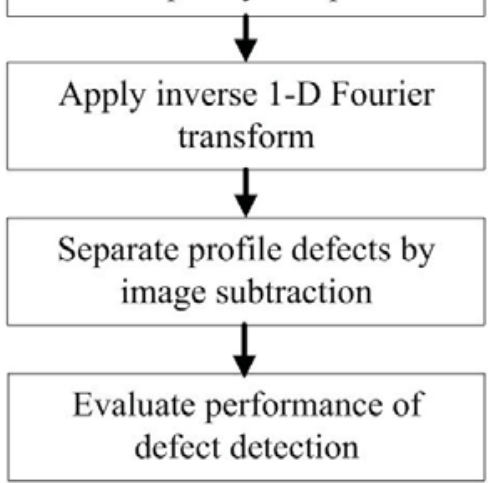

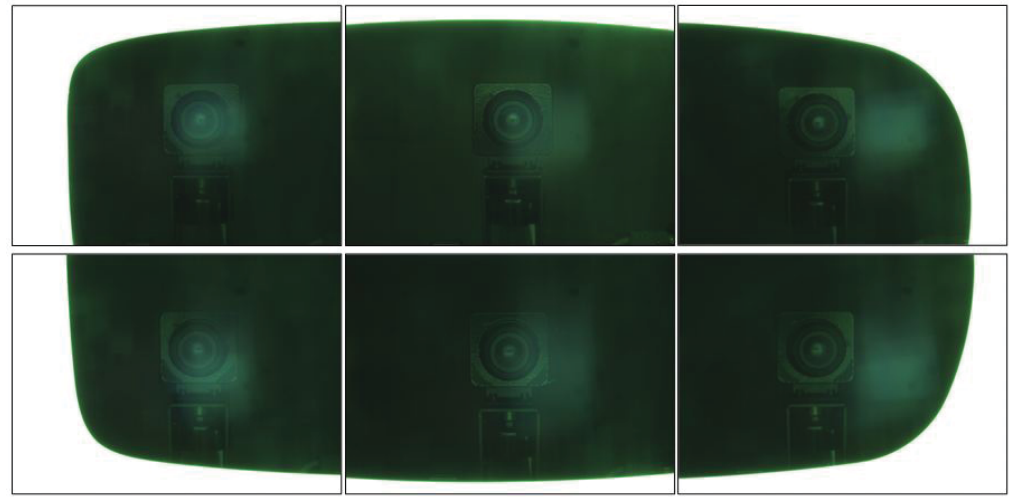

Figure 7. An entire testing sample is equally captured into 6 testing images
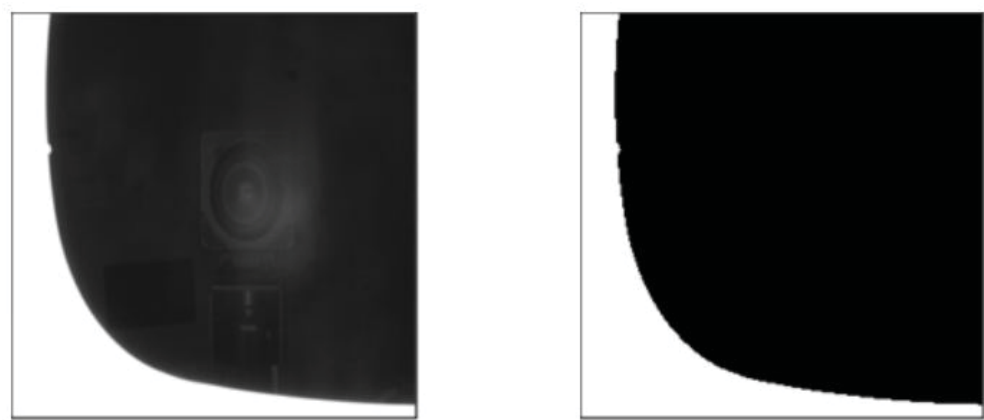

(b)

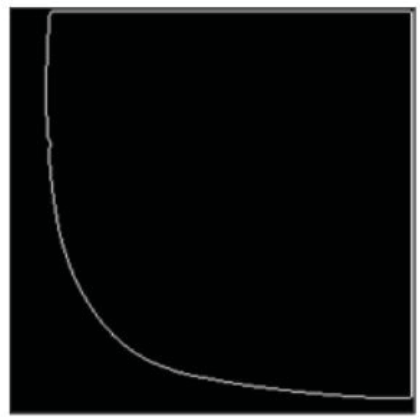

(c)

Figure 8: Results in the process of image binarization and edge detection: (a) primitive image,

(b) binary image, (c) edge image 
the mirror shapes can be estimated by a little number of frequencies, FDs are used to rebuild the shapes of normal mirrors and further identify the defectives.

Let $x(z)$ and $y(z)$ be the coordinates of the $z$-th pixel on the border of a specified 2-D object having $\mathrm{N}$ pixels ( $\mathrm{xi}$, yi) where $\mathrm{i}=0,1,2, \ldots, \mathrm{N}-1$, the complex numbers can be formed as $s(z)=x(z)+j y(z)$ :

$s(z)=x(z)+j y(z), z=0,1,2, \ldots, N-1$

where $\mathrm{j}=\sqrt{ }(-1)$. The FDs of this object are defined as the $1-D$ discrete Fourier transform of $s(z)$ :

$$
a(v)=\frac{1}{N} \sum_{z=0}^{N-1} s(z) e^{-j 2 \pi v z / N}, v=0,1,2, \ldots, N-1
$$

Since the discrete Fourier transform is reversible, all the details regarding the object is included in the FDs. The primitive shape can be restored from the reverse Fourier transform:

$$
s(z)=\sum^{N-1} a(v) e^{j 2 \pi v z / N}, z=0,1,2, \ldots, N-1
$$

If merely some coefficients of the reverse are utilized, the border turns into simple. We can adopt $\mathrm{P}<\mathrm{N}$ FDs correlated to the low-frequency coefficients of the border to depict the 2-D object. The rebuilt shape on the basis of these FDs approximates the object without the high-frequency coefficients:

$\hat{s}(k)=\sum_{u=0}^{p-1} a(u) e^{j 2 \pi u k / N}, k=0,1,2, \ldots N-1$

It seems that the rebuilt images utilizing a few percentage of the low-frequency coefficients are highly close to the primitive image, which can be rebuilt utilizing a full percentage of the FDs. This is because the low-frequency coefficients offer almost all of the messages regarding a shape. High-frequency coefficients are simply influenced by noise and merely stand for trivial details, often with little value to shape recognition.

After the edge coordinates of a shape is transformed to 1-D Fourier descriptor domain, the low-frequency coefficients are located in the middle of the series and the neighborhood is the recovery interval to be retained. After retaining the portion of $\boldsymbol{P}$ low-frequency coefficients in the middle and assign the other coefficients to zero, we take reverse Fourier transform to obtain the approximated coordinates of the primitive shape. The selected recovery points are denoted as:

$$
a(v)= \begin{cases}v_{i} & \text { if }((K-P) / 2)<i \leq(K-P+1) \\ 0 & \text { otherwise }\end{cases}
$$

where $\boldsymbol{K}$ is the total edge points, $\boldsymbol{P}$ is the number of recovery points, $\boldsymbol{a}$ is the index of 1-D Fourier descriptors. Figure 9 shows how the recovery points are selected in the Fourier descriptors.

The lower-frequency FDs have information regarding the widespread shape, and the higher-frequency FDs have information regarding the trivial details. Since the Fourier transform has a complex-valued transform, the frequency domain has negative-frequency coefficients, posi-

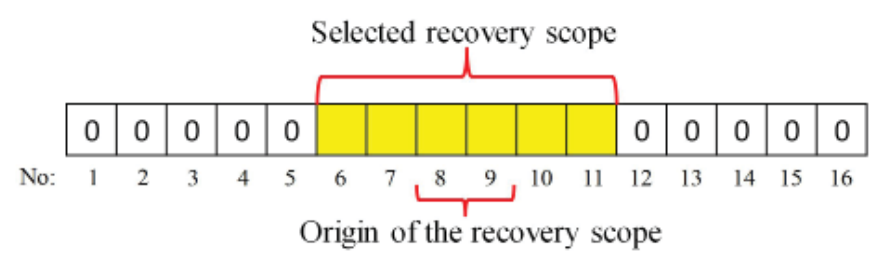

Figure 9: The selection of recovery points in 1-D Fourier descriptors

tive-frequency coefficients, and the DC coefficients in the middle. Consequently, the reverse Fourier transform with $\mathrm{P}<\mathrm{N}$ coefficients requires to include both positive and negative components. When an object has protruding or dent parts, the FD can rebuild an approximated shape of the object without a bumpy surface by selecting the proper recovery parameters to retain low-frequency components.

By recovering a few low-frequency coefficients, the Fourier descriptor can reconstruct a resemble shape of the object boundary. Since the edge point number of testing objects are distinct in applications of industrial inspection, we select a fixed percentage of the total number of edge points as the recovery points. The fixed percentage is called retaining rate $\mathrm{Pr}$ and the number of recovery points is determined as:

$P=K \times P r$

In general, the Pr values within 5\% 10\% can rebuild an approximated shape of a boundary [23]. If an object with a large indentation needs to be recovered, a less retaining rate will be selected.

Only a few low-frequency coefficients are adequate to depict and rebuild the contour of boundary nearly. However, if these high-frequency coefficients are straight eliminated for boundary compression, the rebuilt segment will tend to be closed and result in extreme distortions. In the ordinary use of FDs, the discrete Fourier transform is directly conduct on the entire border sequence, whereas under small retaining rates, contents such as corners and acute angles will be removed.

The object boundaries in the rebuilt image are easy to be over-smoothed or under-smoothed if an inadequate retaining rate is chosen in the Fourier descriptors method. Thus, it is vital to determine how many low-frequency coefficients need to be chosen and applied in the rebuilt process. With more coefficients, the object resembles the primitive. With fewer coefficients, the object turns smoother and rounder. Figure 10 shows the rebuilt effects of object contours for different retaining rates Pr. Apparently, the rebuilt shapes utilizing a few percentage of the low-frequency coefficients are quite close to the real shape, which can be perfectly rebuilt utilizing all of the FDs. However, if too little low-frequency coefficients of the reverse transform are utilized, the border turns into simple.

After an enough portion of the low-frequency coefficients are utilized to rebuild the object, we obtain a figure very close to the real shape of the object. Figure 11 shows the rebuilt process of an object contour image. We apply 


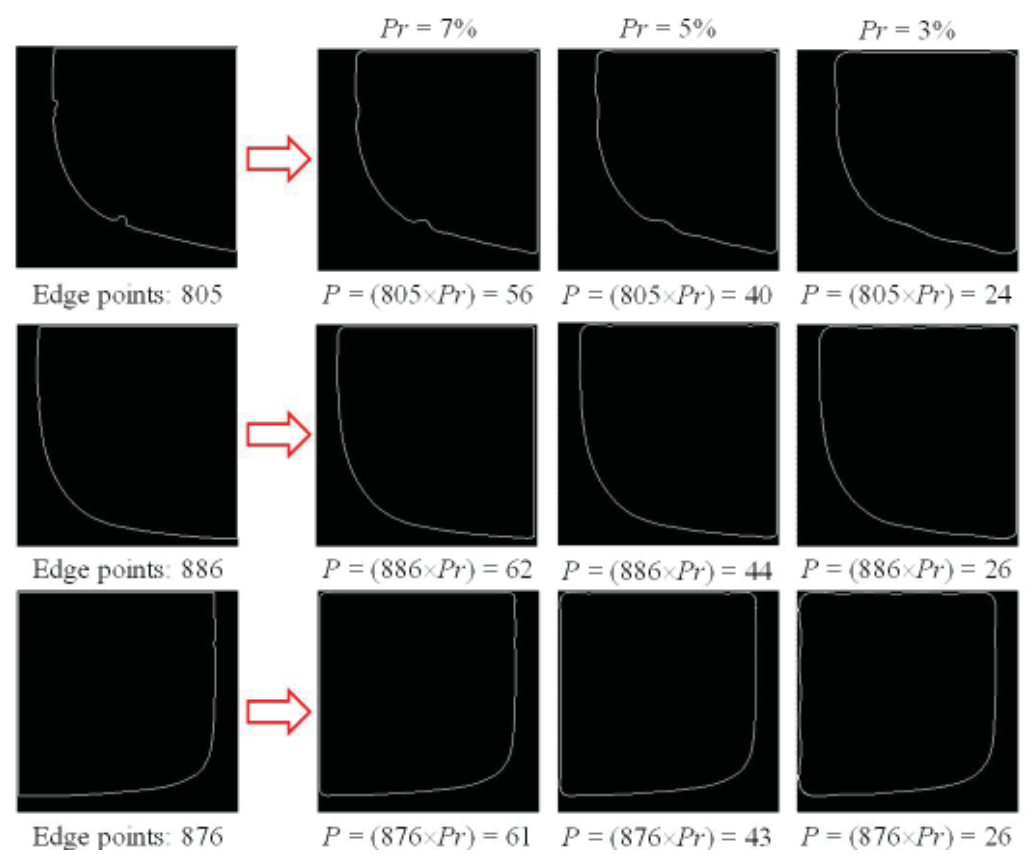

Figure 10: Rebuilt effects of object contours for different retaining rates $\mathrm{Pr}$

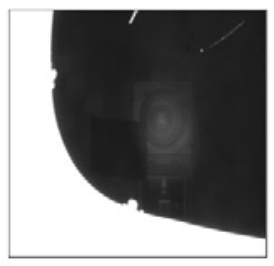

(a) Original image

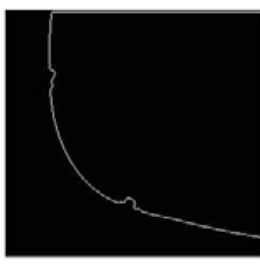

(b) Edge image

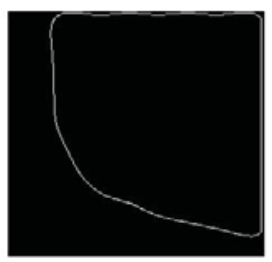

(c) Rebuilt edge image

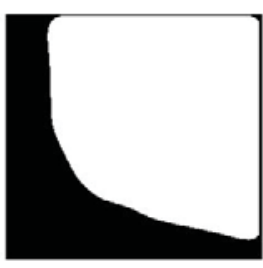

(d) Rebuilt contour image

Figure 11: Rebuilt process of an object contour image

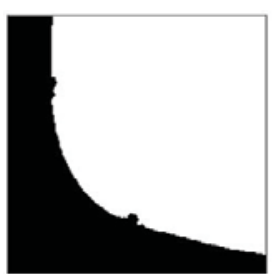

(a) Binary contour image

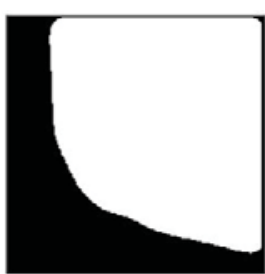

(b) Rebuilt contour image

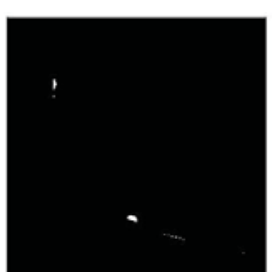

(c) Difference image

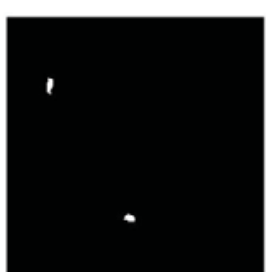

(d) Ground truth image

Figure 12: Comparison of image differences between binary contour image and rebuilt contour image

Sobel edge detector to the binary image for obtaining the edge information of the object and extract the contour information of the edge image by Fourier descriptors. By choosing a suitable retaining rate, the low-frequency components are recovered to rebuild the object edge and obtain the rebuilt contour image for further comparison of image differences.

For comparing the differences between the binary contour image $(\operatorname{im}(x, y))$ and the rebuilt contour image $(\operatorname{rim}(x, y))$, we use image subtraction to obtain the resulting difference image $(\mathrm{R}(\mathrm{x}, \mathrm{y}))$ expressed as,

$R(x, y)=\operatorname{rim}(x, y)-i m(x, y)$

After the two images are subtracted, the remaining parts in the difference image are the inspected profile flaws. Figure 12 displays the comparison of image differences.

\section{EXPERIMENTS AND DISCUSSION}

To strengthen the visibility of object edges on surfaces of car mirrors in the stage of image acquisition, we make use of the following equipment in the developed vision system: a white LED backlight plate with size of $300 \times 300 \mathrm{~mm}$, a 500M-pixels color CCD, a lens with focal length $12 \mathrm{~mm}$, and a three-axis XYZ electronic controlled table. To clearly acquire the damaged edges on each mirror, six images are equally captured for a testing sample. Figure 13 shows the schematic diagrams (top view and side view) of image acquisition for a testing mirror. Experiments are conducted on 80 real car mirrors (30 normal mirrors and 50 defective mirrors) to assess the manifestation of the suggested scheme. Each image of a partial car mirror has a dimension of 256 by 256 pix 


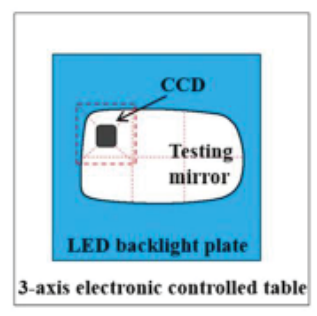

(a) Top view
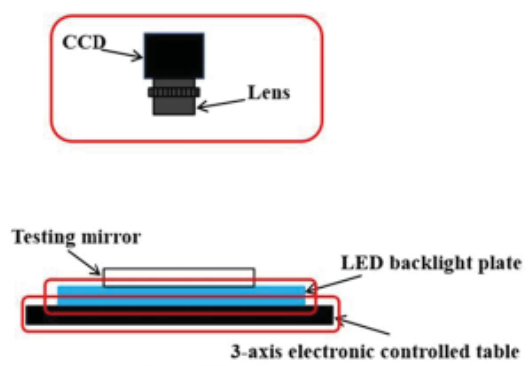

(b) Side view
Figure 13: Schematic diagrams of image acquisition for a testing mirror

els with an intensity of 8 bits. This profile flaw inspection arithmetic is implemented in $\mathrm{C}++$ programming language and conducted on the 6th edition of $\mathrm{C}++$ Builder compiler on a desktop computer (Intel Core i5-4210M CPU @ 2.60G $2.60 \mathrm{GHz}$ ).

To quantitatively confirm the capability of the recommended approach, we compare the outcomes of these assessments against those provided by technical assessors (i.e. ground truth). The manifestation evaluation measures, $(1-\alpha)$ and $(1-\beta)$, are adopted to stand for exact inspection decisions; the larger the two measures, the more exact the inspection results. Incorrect alert rate ( $\alpha$, regarding normal districts as flaws), divide the area of regular district inspected as flaws by the area of real regular district to get the mistake. Absent alert rate $(\beta$, failing to alert real flaws), divide the area of undetected real flaws by the area of real flaws to get the mistake. For the two measures, the smaller the measure value, the better the inspection outcome [26].

Figure 14 shows a user interface layout of the implemented profile flaw inspection system. The interface layout depicts the results and differences conducted by the recommended method of Fourier descriptors in various stages for detecting profile flaws in appearances of car mirrors. The image (1) is a captured image from a portion of a testing sample. The image (2) is a binary image of applying the Otsu method to the testing image and the image (3) addresses the mirror profile after the Sobel edge detectors are executed. The image (4) is a reconstructed profile image after some low-frequency coefficients specified by the assigned retaining rate were recovered in Fourier domain and then the reverse Fourier transform was taken. The image (5) is the corresponding reconstructed binary image of image (4). The image (6) reveals that the profile flaws in the mirror appearance are exactly identified in a binary image, no matter of thecurved surfaces and high reflection.

Selection of the key parameter $\mathrm{Pr}$, retaining rate, in the FDs method will significantly influence the inspection results of the damaged edges. To assess the influence of changing various retaining rates after the procedure of reverse Fourier transform, experiments present the performance indices of detection results by $\mathrm{Pr}$ from the range 3.3 to 3.8. Figure 15 shows locations of the two paired indices $(\alpha, 1-\beta)$ on the ROC (Receiver Operating Curve) plane [27]. The size of retaining rate 3.5 is more appropriate to stress flaws inspected in this rebuilt image. The tests on a diversity of original images have verified that retaining rate 3.5 achieves good satisfactory points on the ROC plane for this profile defect inspection application. Figure 16 depicts the outcomes of flaw inspection by setting the retaining rate $\mathrm{Pr}=3.5$.

One known scheme regularly applied to shape description is compared with the proposed approach to contrastthe effects of profile flaw inspection. To demonstrate the flaw inspection outcomes of a whole testing sample, Figure 17 demonstrates fractional outcomes of inspecting profile flaws by the curvature method [13], the proposed

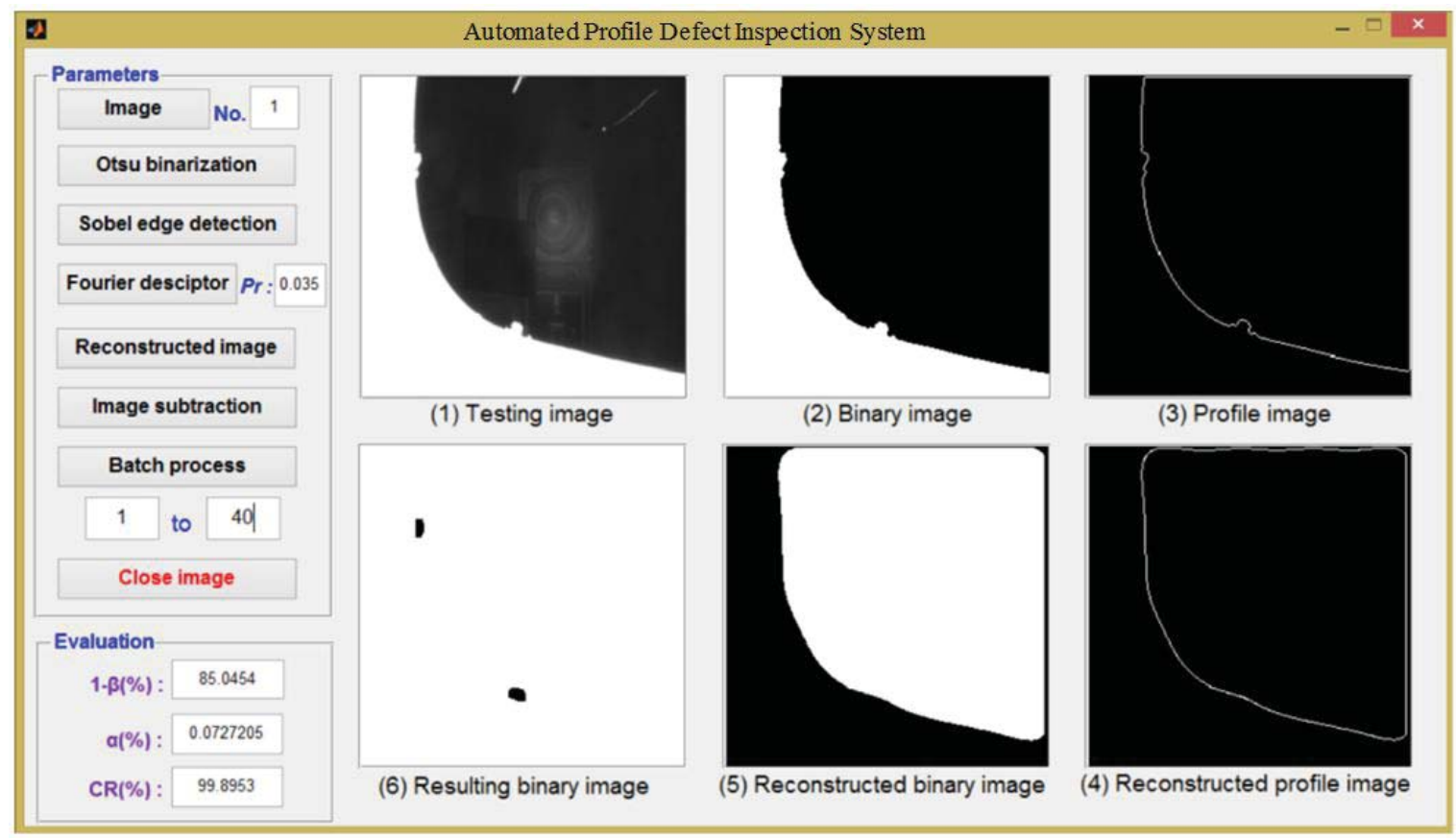

Figure 14: A user interface layout of the automated profile flaw inspection system 


\begin{tabular}{|c|c|c|c|c|c|c|}
\hline $\operatorname{Pr}(\%)$ & 3.8 & 3.7 & 3.6 & 3.5 & 3.4 & 3.3 \\
\hline$\alpha(\%)$ & 0.070 & 0.073 & 0.074 & 0.073 & 0.067 & 0.069 \\
\hline $1-\beta(\%)$ & 76.738 & 82.335 & 84.833 & 85.045 & 79.871 & 77.796 \\
\hline
\end{tabular}

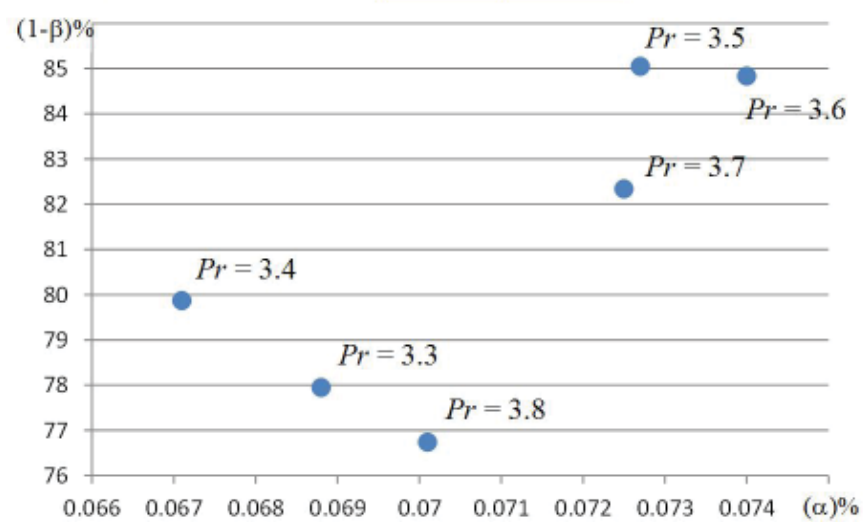

Figure 15: Various locations of the two paired indices $(\alpha, 1-\beta)$ for the retaining rates from range 3.3 to 3.8 on the ROC plane
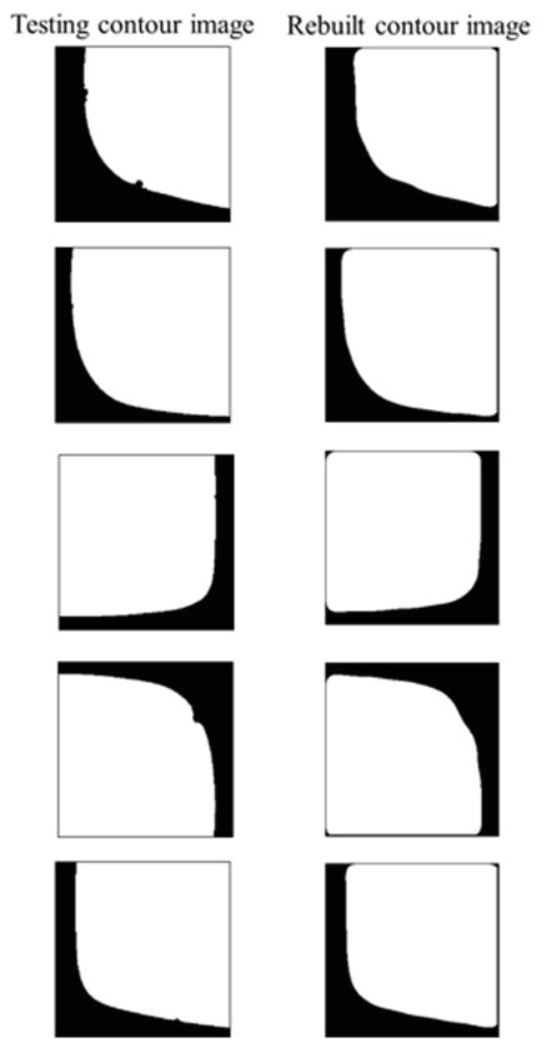

Figure 16: Outcomes of profile flaw detection by applying retaining rate Pr=3.5 in FDs method
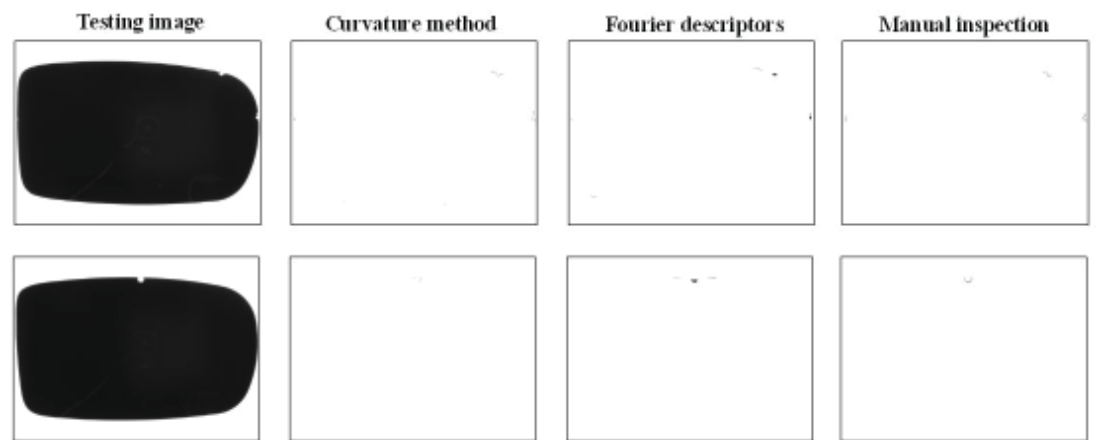

Figure 17: Resulting examples of two whole testing samples for car mirror flaw inspection by curvature method, suggested method, and manual inspection

approach, and the manual inspection, separately. The curvature method makes lots of wrong decisions (absent alerts) on profile flaw inspection. The suggested method inspects almost all of the profile flaws and make less wrong decisions. Therefore, the suggested technique excels the curvature method in the profile flaw inspection of car mirrors with high reflection and curved surfaces.

Table 2 shows the compared effects of profile flaw detection outcomes in the executed tests. One spatial domain technique and one frequency domain skill are evaluated against the results by professional assessors. The means of flaw inspection rates $(1-\beta)$ of all testing trials by the two techniques are, $66.44 \%$ by curvature method and $85.05 \%$ by the proposed approach. Nevertheless, the spatial domain technique has very larger incorrect alert rate $(\alpha), 0.48 \%$ by curvature scheme. Contrarily, the frequency domain skill has quite smaller incorrect alert rate, $0.07 \%$ by proposed approach. The proposed approach has a larger correct classification rate, $97.47 \%$,
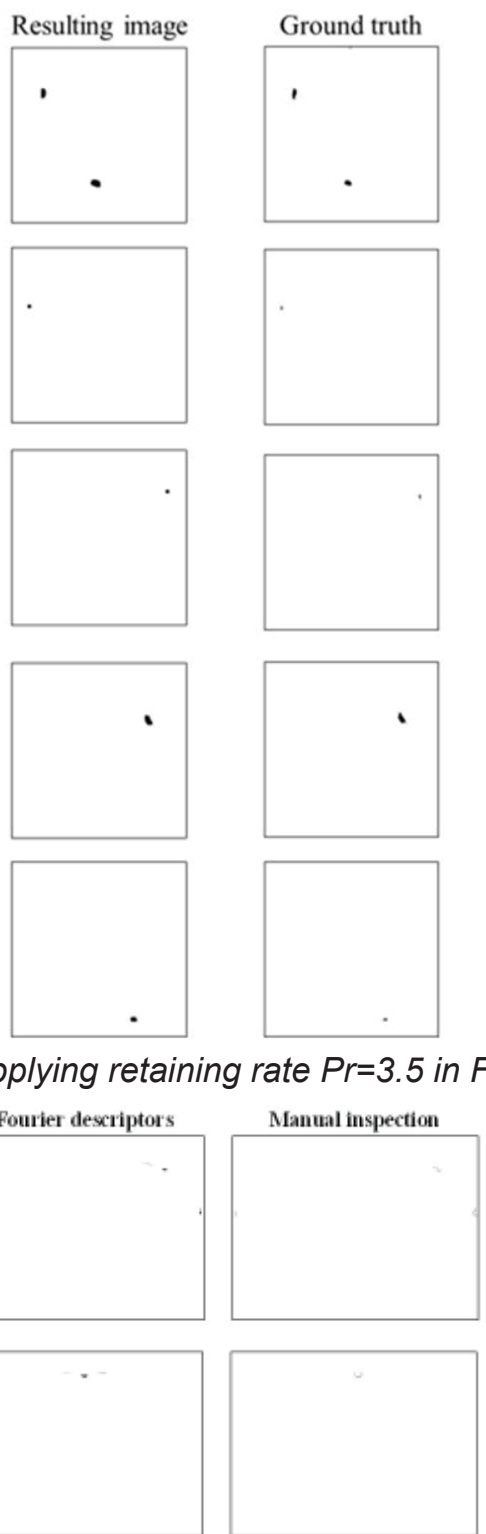
Table 2: Compared effects table of two flaw detection approaches

\begin{tabular}{|l|c|c|c|c|}
\hline $\begin{array}{l}\text { Inspection } \\
\text { schemes }\end{array}$ & $\begin{array}{l}\text { Incorrect alert } \\
\text { rate } \alpha(\%)\end{array}$ & $\begin{array}{l}\text { Flaw } \\
\text { inspection } \\
\text { rate 1- } \beta(\%)\end{array}$ & $\begin{array}{l}\text { Correct classification } \\
\text { rate (\%) }\end{array}$ & $\begin{array}{l}\text { Execution } \\
\text { time (sec) }\end{array}$ \\
\hline $\begin{array}{l}\text { Curvature } \\
\text { method }\end{array}$ & 0.48 & 66.44 & 88.56 & 0.18 \\
\hline $\begin{array}{l}\text { Suggested } \\
\text { method }\end{array}$ & 0.07 & 85.05 & 97.47 & 0.32 \\
\hline
\end{tabular}

than does the curvature scheme, $88.56 \%$, applied to profile flaw detection of car mirrors. More specifically, the recommended approach not only has a larger flaw inspection rate but also has a smaller incorrect alert rate utilized to inspect the profile flaws on car mirror images. The means of execution time for dealing with an image of size 256 by 256 pixels are as following: 0.18 seconds by the curvature method and 0.32 seconds by the suggested approach. The mean execution time of the suggested approach is almost two times longer than that of the curvature method, but it is acceptable for practical implementation of an automated visual inspection system. The proposed approach surmounts the troubles of inspecting profile flaws on curved surfaces of car mirrors with high reflection and overbears in its ability of exactly distinguishing profile flaws from normal districts.

\section{CONCLUSION}

This research applies Fourier transform based shape descriptor technique to the automatic inspection of profile defects in uneven contours of car mirrors. This Fourier descriptors scheme achieves self-comparison between the testing image and the rebuilt one and does not require feature extraction and shape matching procedures. The suggested approach does not rely on any standard pattern for comparison and it is without the part positioning problem. Experimental results demonstrate that the proposed method reaches a large $85.05 \%$ probability of exactly distinguishing profile defects from rough boundaries and a small $0.07 \%$ probability of wrongly inspecting normal districts as profile flaws on uneven contours of car mirrors. Two limitations of the current method need to be conquered in the further study, it will make lots of absent alerts if the profile defects are clustering in a narrow range, and it is not sensitive to distinguish the profile defects with gradual change in shapes.

\section{ACKNOWLEDGEMENTS}

This study was partially sponsored by the Ministry of Science and Technology, Taiwan, for the financial support through the Grant MOST 105-2221-E-324-013-MY2.

\section{REFERENCES}

1. Huang, S.H., Pan, Y.C. (2015). Automated visual inspection in the semiconductor industry: A survey. Computer in Industry, 66, 1-10.
2. Neogi, N., Mohanta, D.K., Dutta, P.K. (2014). Review of vision-based steel surface inspection systems. EURASIP Journal on Image and Video Processing, 50(1), 1-19.

3. Lin, H.D., Chiu, S.W. (2011). Automated surface micro flaw inspection for quality control of electronic chips.International Journal of the Physical Sciences, 6(23), 5528-5539.

4. Lin, H., Li, B., Wang, X.G., Shu, Y.F., Niu, S.L. (2019). Automated defect inspection of LED chip using deep convolutional neural network. Journal of Intelligent Manufacturing, 30(6), 2525-2534.

5. Park, J.K., Kwon, B.K., Park, J.H., Kang, D.J. (2016). Machine learning-based imaging system for surface defect inspection.International Journal of Precision Engineering and Manufacturing-Green Technology, 3(3), 303-310.

6. Li, D., Liang, L.Q., Zhang, W.J. (2014). Defect inspection and extraction of the mobile phone cover glass based on the principal components analysis. International Journal of Advanced Manufacturing Technology, 73, 1605-1614.

7. Lin, H.D., Tsai, H.H. (2012). Automated quality inspection of surface defects on touch panels. Journal of the Chinese Institute of Industrial Engineers, 29(5), 291-302.

8. Yuan-Shyi, C. P., \& Lin, H. [2018]. Creation of image models for inspecting visual flaws on capacitive touch screens. Journal of Applied Engineering Science, 16(3), 333-342.

9. Lin, H.D., Chiu, Y.S.P.(2012). Automated flaw detection for lens components. Advanced Science Letters, $17,114-121$.

10. Lin, H., \& Chen, H. [2018]. Automated visual fault inspection of optical elements using machine vision technologies. Journal of Applied Engineering Science, 16(4), 447-453.

11. Chiu, Y.P., Lo, Y.C., Lin, H.D. (2017). Hough transform based approach for surface distortion flaw detection on transparent glass. International Journal of Applied Engineering Research, 12(19), 8150-8159.

12. Lin, H.D., Hsieh, K.S. (2018). Detection of surface variations on curved mirrors of vehicles using slight deviation control techniques. International Journal of Innovative Computing Information and Control, 14(4), 1407-1421. 
13. Gonzalez, R.C., Woods, R.E. (2008). Digital Image Processing. 3rd Ed., Prentice Hall, New Jersey, USA.

14. Dawari, V.B., Vesmawala, G.R. (2013). Modal curvature and modal flexibility methods for honeycomb damage identification in reinforced concrete beams. Procedia Engineering, 51, 119-124.

15. Samet, A., Hui, Y., Souf, M.A.B., Bareille, O., Ichchou, M., Fakhfakh, T., Haddar, M. (2019). Experimental investigation of damage detection in platelike structure using combined energetic approaches. Proceedings of the Institution of Mechanical Engineers, Part C: Journal of Mechanical Engineering Science, 233(4), 1193-1203.

16. Liu, J., Shi, Z., Shao, Y. (2017). An investigation of a detection method for a subsurface crack in the outer race of a cylindrical roller bearing. Eksploatacja I Niezawodnosc - Maintenance and Reliability, 19(2), 211-219.

17. Khoje, S., Bodhe, S. (2012). Performance comparison of Fourier transform and its derivatives as shape descriptors for mango grading. International Journal of Computer Applications, 53(3), 17-22.

18. Burla, A., Haist, T., Lyda, W., Osten, W. (2011). Fourier descriptors for defect indication in a multiscale and multisensor measurement system. Optical Engineering, 50(4), 043603.

19. Zheng, Y., Guo, B., Chen, Z., Li, C. (2019). A Fourier descriptor of $2 \mathrm{D}$ shapes based on multiscale centroid contour distances used in object recognition in remote sensing images, Sensors, 19(486), 1-19.

20. Zhang, D., Lu, G. (2002). Shape-based image retrieval using generic Fourier descriptor. Signal Processing: Image Communication, 17, 825-848.

21. Direkoglu, C., Nixon, M.S. (2011). Shape classification via image-based multiscale descriptions. Pattern Recognition, 44, 2134-2146.
22. Zhang, D., Lu, G. (2004). Review of shape representation and description techniques. Pattern Recognition, 37, 1-19.

23. Tsai, D.M., Su, Y.J. (2009). Non-referential, self-compared shape defect inspection for bond pads with deformed shapes. International Journal of Production Research, 47, 1225-1244.

24. Kunttu, I., Lepisto, L., Rauhamaa, J., Visa, A. (2005). Multiscale Fourier descriptors for defect image retrieval. Pattern Recognition Letters, 27, 123-132.

25. Otsu, N. (1979). A threshold selection method from gray level histogram. IEEE Transactions on Systems, Man and Cybernetics, 9, 62-66.

26. Montgomery, D.C. (2013). Statistical Quality Control - A Modern Introduction, 7th Edition, John Wiley \& Sons, New York, NY, USA.

27. Montgomery, D.C., Runger, G.C. (2007). Applied Statistics and Probability for Engineers. 4th Edition, John Wiley \& Sons, New Jersey, USA.
Paper submitted: 14.08.2019.

Paper accepted: 10.02.2020.

This is an open access article distributed under the CC $B Y-N C-N D 4.0$ terms and conditions. 